\title{
Germanium Nanowire Battery Electrodes with Engineered Surface- Binder Interactions Exhibit Improved Cycle Life and High Energy Density without Fluorinated Additives
}

\section{Supporting Information}

Authors: Elena P. Pandres,,$^{\dagger}$, Jarred Z. Olson, ${ }^{\delta,+}$ Cody W. Schlenker, $*, \varsigma_{\perp}$ and Vincent C. Holmberg*,†,,$\perp$

\section{Affiliations:}

${ }^{\dagger}$ Department of Chemical Engineering, University of Washington, Seattle, Washington 981951750, United States

${ }^{\S}$ Department of Chemistry, University of Washington, Seattle, Washington 98195-1700, United States

${ }^{\mathrm{I}}$ Molecular Engineering \& Sciences Institute, University of Washington, Seattle, Washington 98195-1652, United States

${ }^{\perp}$ Clean Energy Institute, University of Washington, Seattle, Washington 98195-1653, United States

*Correspondence to holmvc@uw.edu and schlenk@uw.edu

Germanium Nanowire Synthesis and Passivation. Dodecanethiol-passivated gold (Au) nanocrystals were prepared in a mixture of deionized water and toluene via the Brust method. ${ }^{1}$ Germanium nanowires were synthesized via SFLS-based growth, and surface functionalized in situ via thermally initiated hydrogermylation with 1 -octene. ${ }^{2}$ In a typical reaction, a cylindrical 10 $\mathrm{mL}$ titanium reaction vessel was transferred into a nitrogen atmosphere glovebox, sealed, and placed in a heating block at $380^{\circ} \mathrm{C}$. Keeping the exit valve closed, the reactor was pressurized to 900 psig using anhydrous toluene. A precursor mixture containing $1.7 \times 10^{-2} \mathrm{mg} / \mathrm{mL} \mathrm{Au}$ nanocrystals and $3.3 \times 10^{-2} \mathrm{M}$ diphenylgermane was prepared in anhydrous toluene (385:1 Au:Ge molar ratio). The mixture was loaded into a sealed stainless-steel piston and subsequently injected into the pre-pressurized and pre-heated reactor $\left(900 \mathrm{psig}, 380^{\circ} \mathrm{C}\right)$ at a rate of $0.5 \mathrm{~mL} / \mathrm{min}$ for forty minutes. After nanowire growth, the temperature was then decreased to $220^{\circ} \mathrm{C}$ for surface 
hydrogermylation. Nanowire surface functionalization was carried out by injecting $12 \mathrm{~mL}$ of 2.1 M 1-octene in anhydrous toluene into the reactor at a rate of $0.5 \mathrm{~mL} / \mathrm{min}$ for twenty minutes. The reactor temperature was then sealed and held at $220^{\circ} \mathrm{C}$ for 2 hours, followed by isochoric cooling to room temperature. Nanowires were collected with toluene. All germanium nanowires used in this study were hydrogermylated with 1-octene. Nanowires were washed three times in glass vials with a 2:2:1 ratio of toluene:chloroform:ethanol, and centrifuged at $2355 \mathrm{RCF}$ for ten minutes. The resulting high-aspect-ratio, single-crystalline germanium nanowires produced via this method have diameters ranging from 10 to $100 \mathrm{~nm}$ and lengths ranging from micrometers to millimeters.

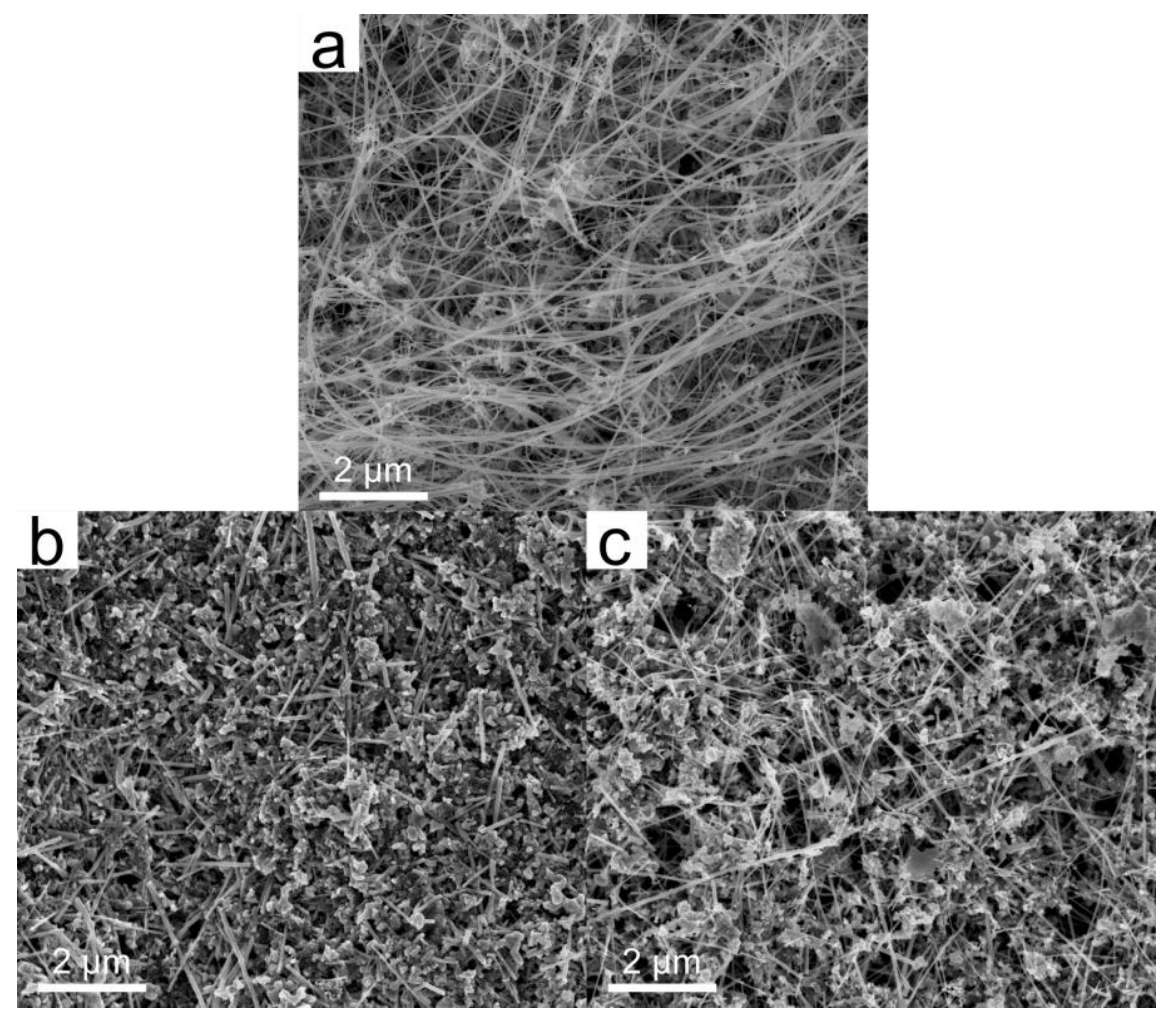

Figure S1. SEM images of (a) 1-octene hydrogermylated Ge nanowires before incorporation into a composite electrode, (b) a composite electrode (70:10:20 mass ratio of Ge NWs:conductive carbon:PAA) fabricated via manual mixing with a mortar and pestle, and (c) a composite electrode (70:10:20 mass ratio of Ge NWs:conductive carbon:PAA) fabricated via magnetic stirring. 


\begin{tabular}{l|cc}
\multicolumn{1}{l}{ Electrode } & $\begin{array}{c}\text { Gravimetric capacity based on } \\
\text { active material mass (mAh/g) }\end{array}$ & $\begin{array}{c}\text { Gravimetric capacity based on } \\
\text { entire electrode mass (mAh/g) }\end{array}$ \\
\hline \hline PAA, M\&P & 1261 & 883 \\
\hline PAA, M\&P, FEC & 1386 & 970 \\
\hline PAA, M\&P, VC & 1182 & 828 \\
\hline
\end{tabular}

Table S1. Gravimetric capacities of the germanium nanowire electrodes shown in Figure 1b, calculated based on the mass of active material (left) and calculated based on the mass of the entire composite electrode (including active material, conductive carbon, and binder). Values shown correspond to the second cycle.

(a)

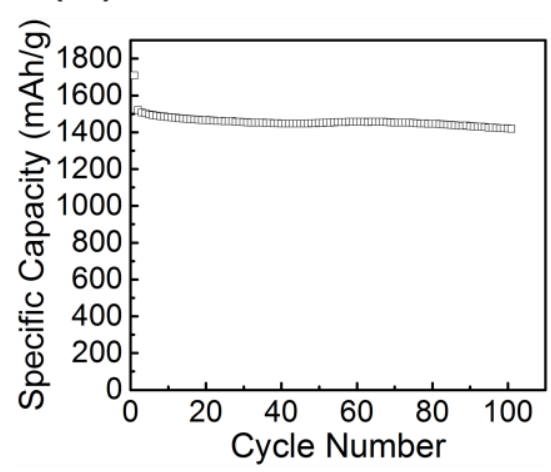

(b)

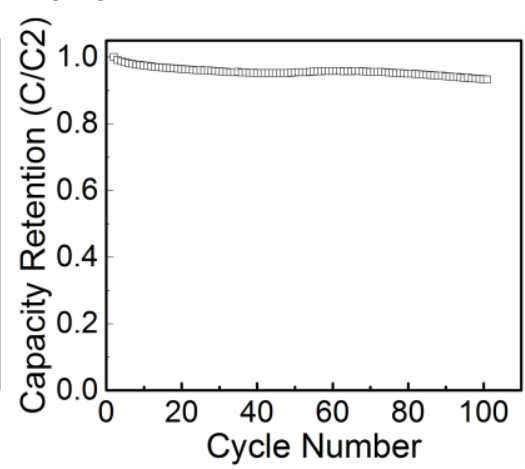

(c)

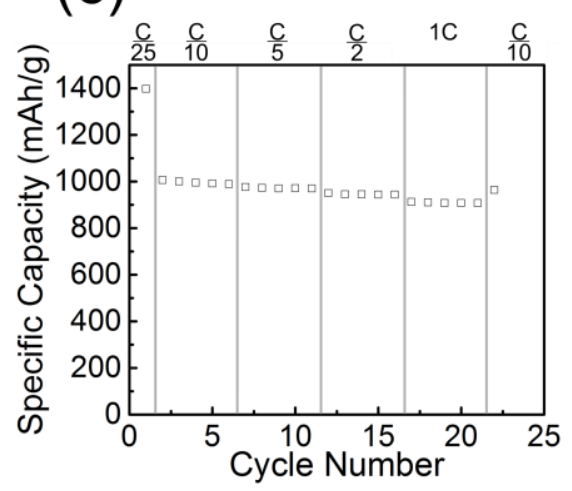

Figure S2. (a) Specific capacity and (b) capacity retention for electrodes cycled at a rate of C/10 $(138 \mathrm{~mA} / \mathrm{g})$ over 100 cycles. (c) Rate test of 1-octene hydrogermylated Ge nanowires cycled at C/25 (55.4 mA/g), C/10 (138 mA/g), C/5 (277 mA/g), C/2 (692 mA/g), 1C (1384 mA/g), and C/10 $(138 \mathrm{~mA} / \mathrm{g})$. Electrodes were fabricated with PAA via manual mixing and were cycled with a $1 \mathrm{M}$ $\mathrm{LiPF}_{6}$ 1:1 w/w EC:DEC electrolyte. 


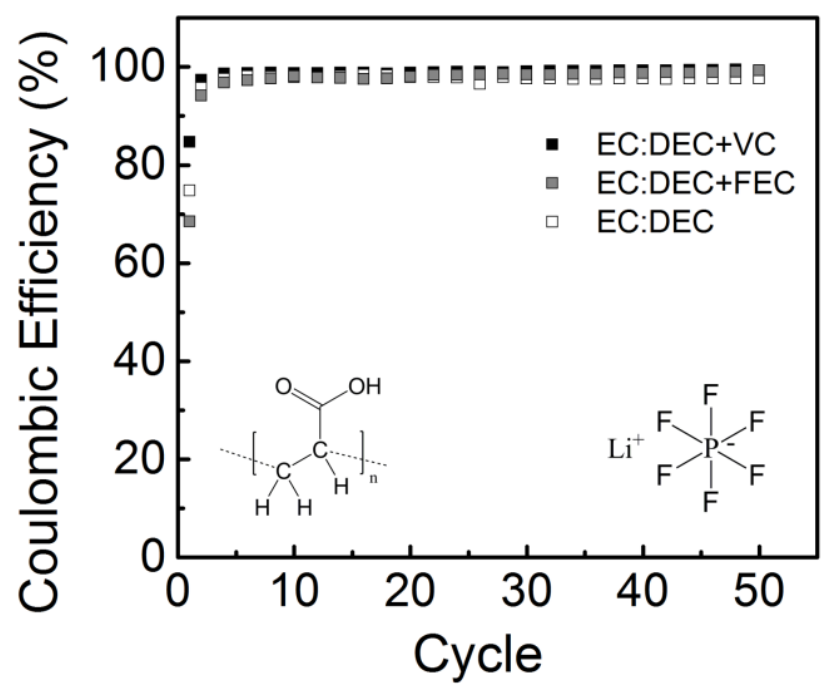

Figure S3. Coulombic efficiencies of germanium nanowire composite electrodes fabricated via manual mixing, using PAA as a binder, and $1 \mathrm{M} \mathrm{LiPF}_{6}$ in 1:1 w/w EC:DEC with different additives: white (no additive), gray (FEC), and black (VC), cycled at a rate of C/10.

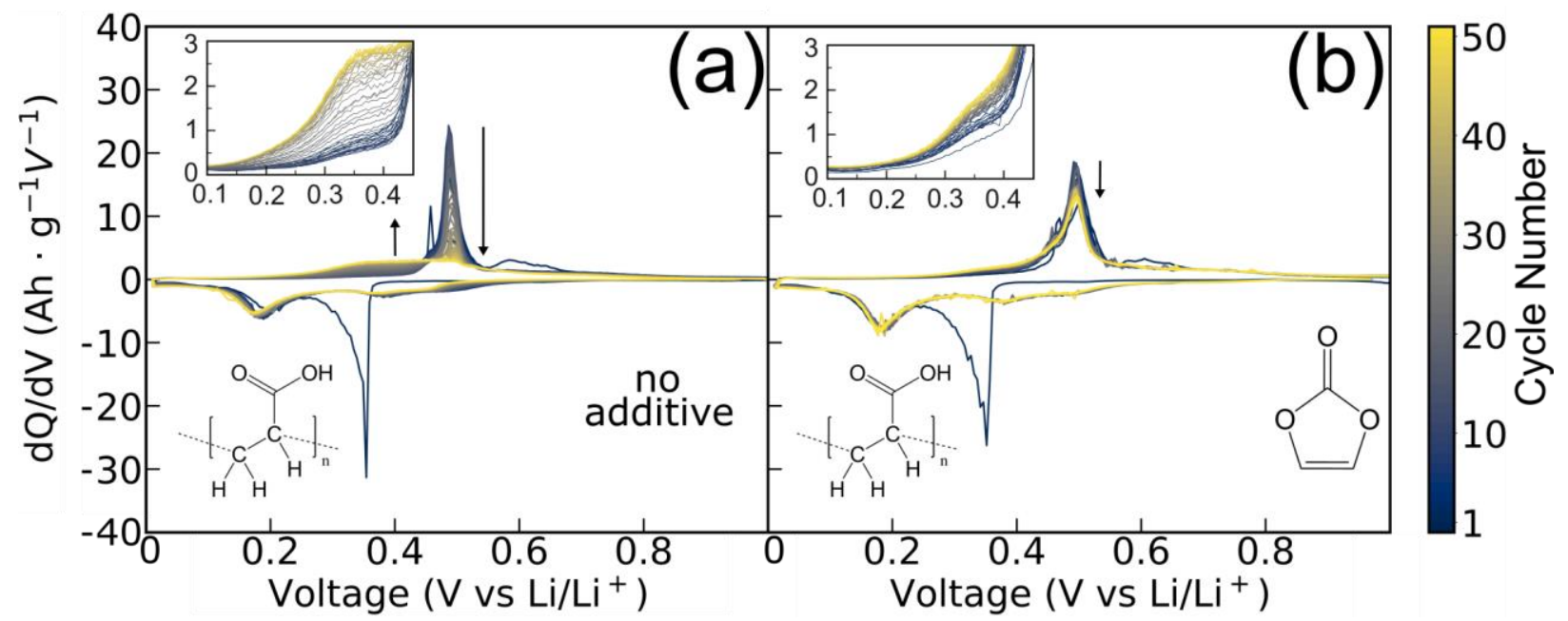

Figure S4. Total differential capacity plots of 1-octene hydrogermylated germanium nanowire composite electrodes that employ PAA as a binder, and $\mathrm{EC} / \mathrm{DEC}+1 \mathrm{M} \mathrm{LiClO}_{4}$ as an electrolyte with (a) no electrolyte additive and (b) VC additive included. The color scale at the rightmost side delineates the evolution of the differential capacity profile over the first 50 cycles for each device. Arrows highlight changes in peak intensity. 


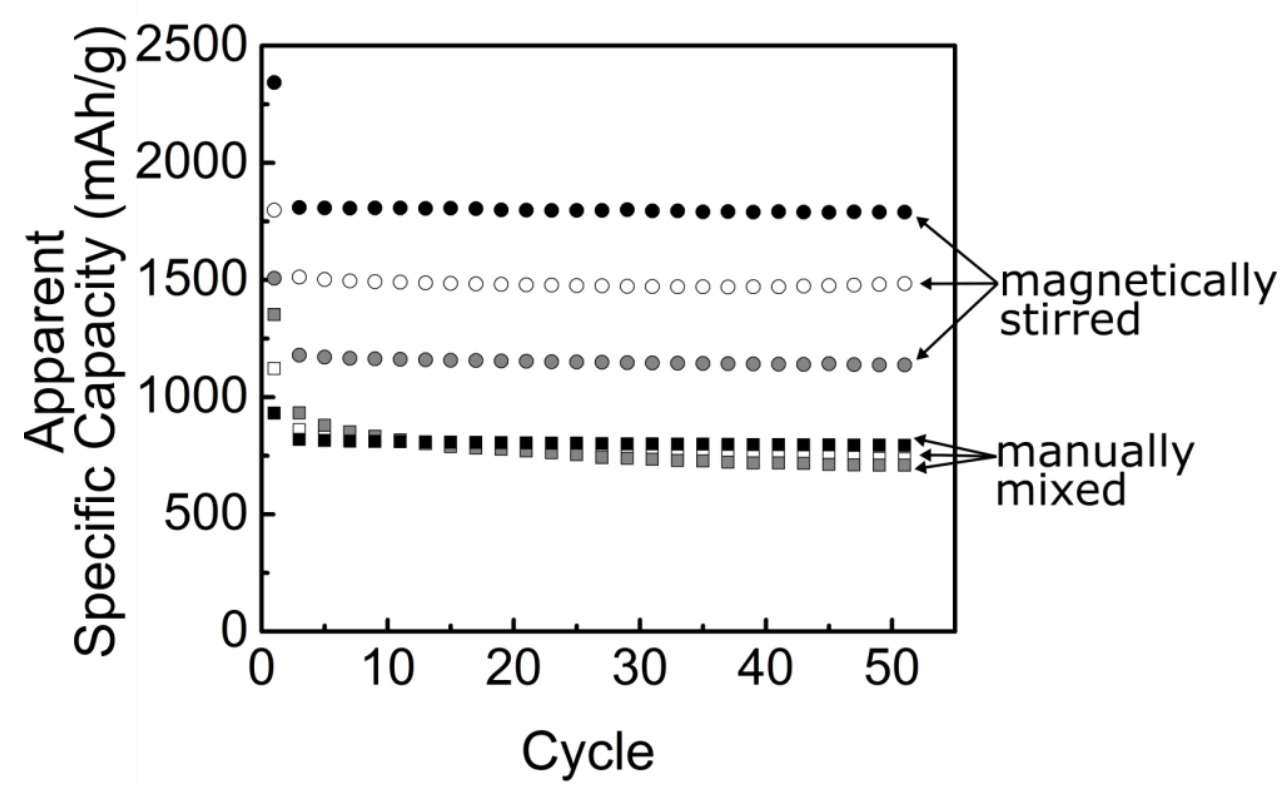

Figure S5. Variations in the apparent specific capacity of electrodes doctor-bladed from manually mixed (squares) or magnetically stirred (circles) PAA slurries, calculated based on the mass of the entire composite electrode. This highlights the severe level of processing variability that exists for electrodes cast from magnetically stirred PAA slurries that are prone to flocculation. White, gray, and black symbols correspond to devices without additive, with FEC, and with VC, respectively.

Note that Figure S5 (above) has been included in order to emphasize the significant processing challenges that exist with respect to producing electrodes with consistent active material loadings using magnetically stirred PAA slurries, resulting in large variations in apparent specific capacity and unreliable gravimetric capacity values. This is supported by the observation that magnetically stirred PAA slurries exhibited increased tendencies toward nanowire flocculation with time. Consequently, we have focused on presenting trends in capacity retention throughout the manuscript, as opposed to specific gravimetric capacity values. 


\begin{tabular}{l|c}
\multicolumn{1}{l}{ Electrode } & $\begin{array}{c}\text { Gravimetric capacity based on } \\
\text { entire electrode mass (mAh/g) }\end{array}$ \\
\hline \hline PAA, M\&P & $880 \pm 120$ \\
\hline PAA, M\&P, FEC & $970 \pm 75$ \\
\hline PAA, M\&P, VC & $830 \pm 130$ \\
\hline perchlorate, M\&P & $890 \pm 150$ \\
\hline perchlorate, M\&P, VC & $1040 \pm 130$ \\
\hline PAA, stir & $1520 \pm 310$ \\
\hline PAA, stir, FEC & $1190 \pm 170$ \\
\hline PAA, stir, VC & $1800 \pm 1200$ \\
\hline PVDF, M\&P & $270 \pm 20$ \\
\hline PVDF, M\&P, FEC & $570 \pm 40$ \\
\hline PVDF, stir & $700 \pm 70$ \\
\hline PVDF, stir, FEC & $720 \pm 70$ \\
\hline PVDF, stir, VC & $510 \pm 80$ \\
\hline
\end{tabular}

Table S2. Gravimetric capacities of each germanium nanowire composite electrode calculated based on the mass of the entire composite electrode (including active material, conductive carbon, and binder). Values shown correspond to the second cycle. Error bars correspond to the uncertainty in mass loading due to variations in thickness of the copper foil current collector. The manufacturer (MTI) reports copper thicknesses of $10.5 \pm 1.5 \mu \mathrm{m}$, and we measure mass values of $16.16 \pm 0.14$ mg for the copper disc electrodes punched from the foil. The large errors reported for the magnetically stirred PAA electrodes correspond to low mass loadings due to the challenges associated with doctor-blading uniform electrodes from magnetically stirred PAA slurries.

\section{REFERENCES}

1. Brust, M.; Walker, M.; Bethell, D.; Schiffrin, D. J.; Whyman, R., Synthesis of Thiolderivatised Gold Nanoparticals in a Two-phase Liquid-Liquid System. J. Chem. Soc., Chem. Commun. 1994, 7, 801-802.

2. Holmberg, V. C.; Korgel, B. A., Corrosion Resistance of Thiol- and Alkene-Passivated Germanium Nanowires. Chem. Mater. 2010, 22 (12), 3698-3703. 